\title{
How to treat stellar limb darkening in the analysis of microlensing events
}

\author{
David Heyrovský* \\ Institute of Theoretical Physics, Charles University in Prague, Czech Republic \\ E-mail: heyrovsky@utf.mff.cuni.cz
}

\begin{abstract}
A number of caustic-crossing microlensing events have been used to measure limb-darkening coefficients of their source stars. We point out that the usage of analytical limb-darkening laws generally involves two potential problems. First, the tabulated stellar model-atmosphere limbdarkening coefficients are usually computed by a method strongly biased to fitting the stellar limb. Second, even when using less biased coefficients for comparison, the linear law tends to have problems with overall fit quality as well as with flux conservation, and higher-order coefficients can rarely be satisfactorily constrained from light-curve analysis. An alternative approach is to use a "best possible" limb-darkening model directly obtained by principal component analysis (PCA) of model atmospheres. We use a model constructed from Kurucz's full ATLAS9 atmosphere grid, and demonstrate its superior quality in describing limb-darkening profiles. As an example, we analyze the point-lens event OGLE 2004-BLG-254 using the linear law and the PCA limbdarkening model.
\end{abstract}

The Manchester Microlensing Conference: The 12th International Conference and ANGLES Microlensing Workshop

January 21-25, 2008

Manchester, $U K$

${ }^{*}$ Speaker. 


\section{Introduction}

Stellar limb darkening (hereafter LD) has traditionally been of little interest outside the stellar atmosphere modeler community, and its observational relevance has been largely limited to studies of the Sun and eclipsing binaries. By now the range of astrophysical observations capable of resolving stellar LD has expanded, including interferometry of nearby giants and supergiants, extrasolar planetary transits, and caustic-crossing gravitational microlensing events.

The usual approach in the analysis of such observations is to treat LD as a nuisance effect that has to be included in order to obtain other parameters such as stellar or planetary radii, impact parameters, etc. Ideally one should use a theoretical LD profile directly from an appropriate model atmosphere, e.g., from Kurucz's ATLAS9 model grid (Kurucz 1999a, 1993b, 1994). An easier option is to use an approximation given by some simple analytical LD law and take the necessary coefficients from tables precalculated for the model grid. However, one has to pay attention to the sensitivity of such coefficients to the fitting method used in their calculation.

Sufficiently good observations permit another approach by providing the opportunity to observationally constrain or directly measure the star's LD, and thus test the structure of theoretical stellar atmosphere models. This requires a simple LD parametrization, because the observational precision rarely permits more than a single LD parameter to be extracted. At the same time the necessary model should be versatile enough to describe adequately the LD of relevant model atmospheres, which are often poorly approximated by simple analytical laws.

\section{Coefficients of Analytical Limb-darkening Laws}

Analytical LD laws are mostly constructed as linear combinations of simple functions of $\mu=\sqrt{1-r^{2}}$, where $r$ denotes the radial position on the disk. The simplest law is the two-term linear LD, three-term LD laws include quadratic, square-root, and logarithmic LD (see for ex-
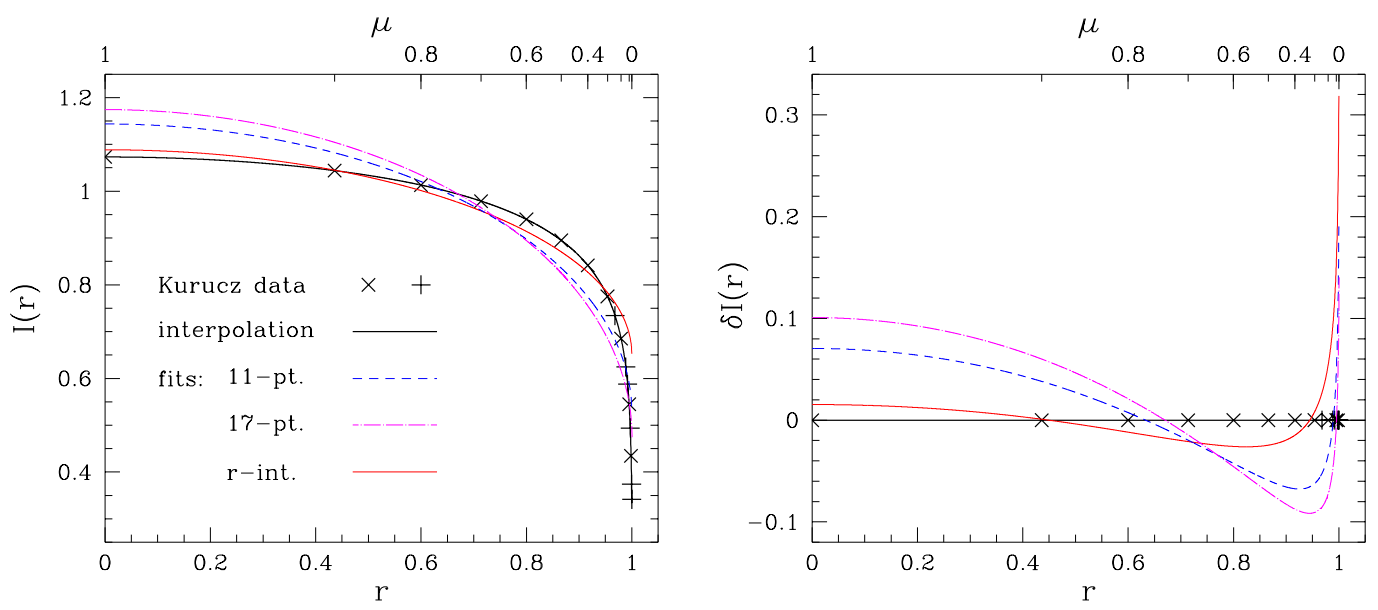

Figure 1: Sample Kurucz ATLAS9 model $V$-band LD profile $\left(T_{\text {eff }}=4250 \mathrm{~K}, \log \mathrm{g}=4.5,[\mathrm{Fe} / \mathrm{H}]=-3.5\right)$ and its linear LD least-squares fits. The 17 data points are marked by crosses (11 points) and plus signs, their spline interpolation by the bold solid line. Fits include the 17 -point (dot-dashed; $u_{17}=0.596$ ), the 11-point (dashed; $u_{11}=0.538$ ), and a fit of the spline by radial integration (thin solid; $u_{r}=0.401$ ). Left panel: intensity. Right panel: absolute residuals of the fits. Values of $\mu$ are marked along the upper axes. 

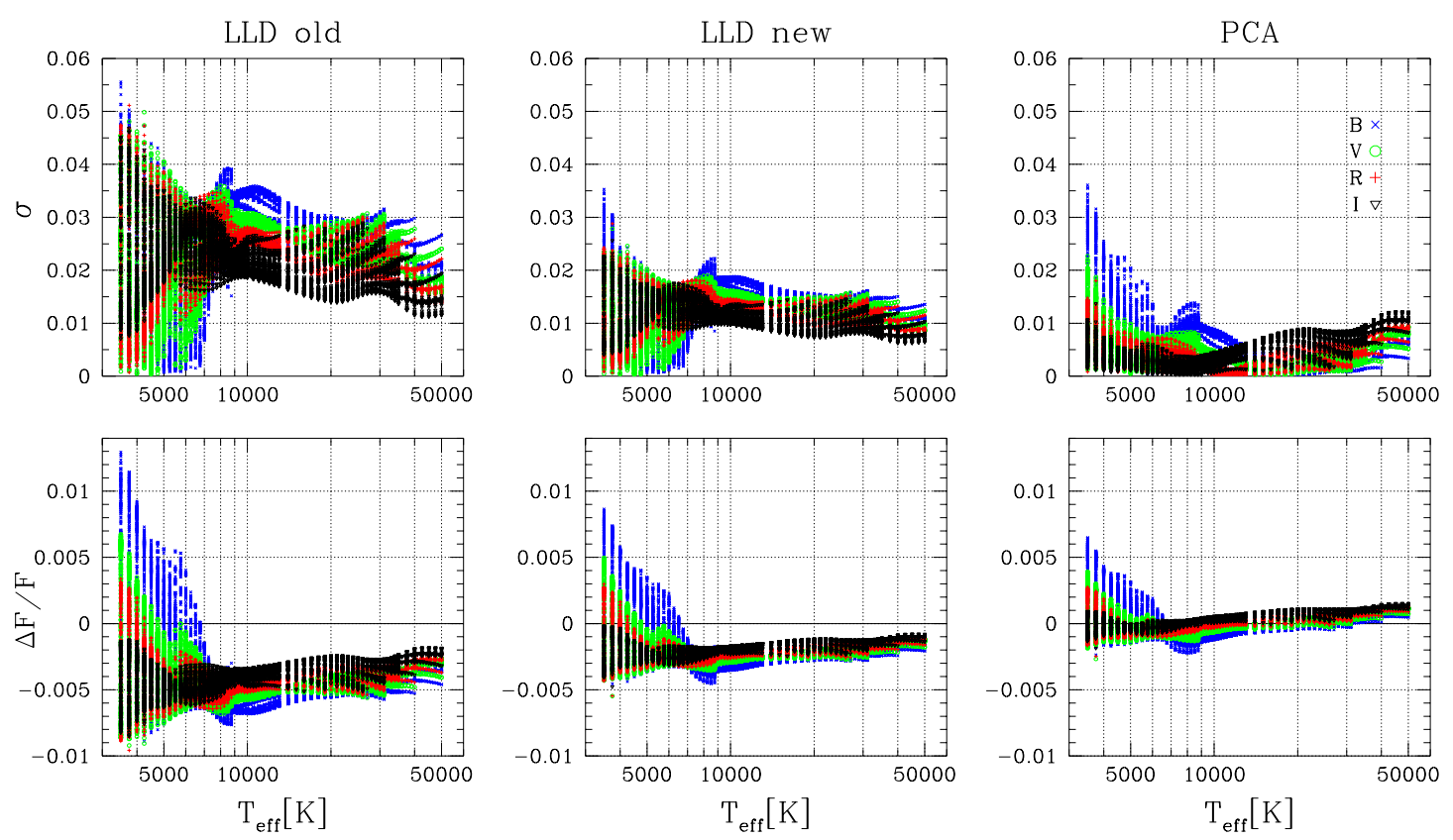

Figure 2: Relative rms intensity residuals $\sigma$ (top) and relative flux excesses $\Delta F / F$ (bottom) for the 11-point linear LD fits (left), $r$-integrated linear fits (center), and PCA LD fits (right) for the Kurucz model grid, plotted as a function of $T_{e f f}$ and color-coded by spectral band (marked in top right panel).

ample Claret 2000). It is well known that the values of LD coefficients determined from model atmospheres are sensitive to the method of their calculation. The difference between methods based on exact flux conservation and those based on least-squares fits of intensity profiles has been discussed by Claret (2000). The former methods lack an explicit requirement for fitting the profile shape, hence the latter are preferrable even though flux is not conserved exactly.

As pointed out by Díaz-Cordovés et al. (1995), even within the latter group the results depend strongly on the particular implementation of least-squares fitting. Figure 1 demonstrates three different fits of the linear law to a sample LD profile of a Kurucz ATLAS9 model (Kurucz 1993b). The first is a direct fit to the 17 Kurucz data points, which include regularly spaced $\mu$ values with extra points close to the limb. To reduce the heavy bias to the limb, the second 11-point fit keeps the regularly spaced values with only a single additional point closer to the limb. This approach has been used for the most widely used tabulated coefficients (e.g., Claret 2000).

Even this fit is clearly biased to the limb, and the fitted profile has a very different shape from the original. The problem lies in the choice of regular spacing of $\mu$ values. This implies for instance that an equal weight is given to the quality of the fit in the inner $20 \%$ of the radial extent as to the outer radial $2 \%$. A better approach is to fit with equal radial weighting. This can be achieved by taking a spline interpolation of the 17 Kurucz points and minimizing the radially integrated squared residual between the spline and the analytical LD law of interest (Heyrovský 2007).

Figure 2 and Table 1 illustrate the improved fit quality in comparison with the commonly used 11-point fit, as measured by the relative rms intensity residual $\sigma$ and the flux excess $\Delta F / F$ of the fit. The computation was performed on BVRI profiles of the full range of Kurucz's ATLAS9 model grid (Kurucz 1993a, 1993b, 1994), including altogether 38,324 LD profiles. In comparison with the 11point fit, the new method improves the average intensity residual and flux excess by a factor of two. Figure 3 demonstrates the difference between linear LD coefficients obtained by the new fitting 


\begin{tabular}{|l|l|cccc|}
\hline LD model & Fitting method & $\begin{array}{c}\text { Average } \sigma \\
(\%)\end{array}$ & $\begin{array}{c}\text { Max. } \sigma \\
(\%)\end{array}$ & Average $|\Delta F / F|$ & Max. $|\Delta F / F|$ \\
\hline \hline Linear & 11-point & 2.344 & 5.554 & $4.38 \times 10^{-3}$ & $1.29 \times 10^{-2}$ \\
& $r$-int. spline & 1.253 & 3.527 & $2.27 \times 10^{-3}$ & $8.65 \times 10^{-3}$ \\
\hline 2-term PCA & projection & 0.479 & 3.609 & $6.55 \times 10^{-4}$ & $6.48 \times 10^{-3}$ \\
\hline \hline Quadratic & 11-point & 0.653 & 1.785 & $1.10 \times 10^{-3}$ & $2.98 \times 10^{-3}$ \\
& $r$-int. spline & 0.417 & 0.991 & $2.35 \times 10^{-4}$ & $6.15 \times 10^{-4}$ \\
\hline Square root & 11-point & 0.288 & 2.115 & $3.32 \times 10^{-4}$ & $4.71 \times 10^{-3}$ \\
& $r$-int. spline & 0.238 & 1.382 & $1.28 \times 10^{-4}$ & $1.99 \times 10^{-3}$ \\
\hline Logarithmic & 11-point & 0.345 & 1.587 & $5.35 \times 10^{-4}$ & $3.41 \times 10^{-3}$ \\
& $r$-int. spline & 0.259 & 0.999 & $1.66 \times 10^{-4}$ & $1.18 \times 10^{-3}$ \\
\hline 3-term PCA & projection & 0.120 & 0.713 & $7.47 \times 10^{-5}$ & $8.60 \times 10^{-4}$ \\
\hline
\end{tabular}

Table 1: Fit quality for different LD models and different least-squares fitting methods. Average and maximum values of the relative rms intensity residual $\sigma$ and the relative flux excess $|\Delta F / F|$, evaluated for $B V R I$ profiles of the full grid of Kurucz model atmospheres.

method and the 11-point method. The differences are substantial and observationally significant, ranging from -0.14 to 0.1 . The old coefficients are most frequently too high, for instance in the $I$ band the new fits are always flatter with coefficients lower than the old values. Heyrovský (2007) demonstrated that the new coefficients are in better agreement with LD measurements for the Sun and a set of eclipsing binaries.

\section{Limb-darkening Model Constructed by Principal Component Analysis}

Even though the new fitting method provides better fits, the average intensity residual for the linear law still remains over $1.2 \%$ and the average flux excess over $0.2 \%$. For many profiles such inaccuracies lead to observationally significant deviations. To construct a better model we look for the best possible LD description in the least possible number of terms. For this purpose we drop the requirement of analyticity and construct such a LD basis numerically by principal component analysis (PCA) of a given set of model atmosphere LD profiles, as described by Heyrovský (2003).

The left panel of Figure 4 illustrates the first three functions of the PCA LD basis constructed using the BVRI profiles of the entire ATLAS9 model grid used above. Already the first function $f_{1}$ has a realistic "mean" LD shape. Each successive added basis function describes ever subtler variations in the LD profile. The basis functions are orthonormal, $\int_{0}^{1} f_{i} f_{j} \mathrm{~d} r=\delta_{i j}$, which means that 1) coefficients of different order are uncorrelated, 2) adding more terms to the model leaves the previous coefficients unchanged, and 3) fitting of any LD profile is performed directly by its projection onto the PCA basis. The two other panels of Figure 4 illustrate examples of bases constructed from a successively narrower parameter range of the Kurucz grid. The full range of LD shapes described by the combination of $f_{1}$ and $f_{2}$ of the PCA basis is shown in the top left panel of 

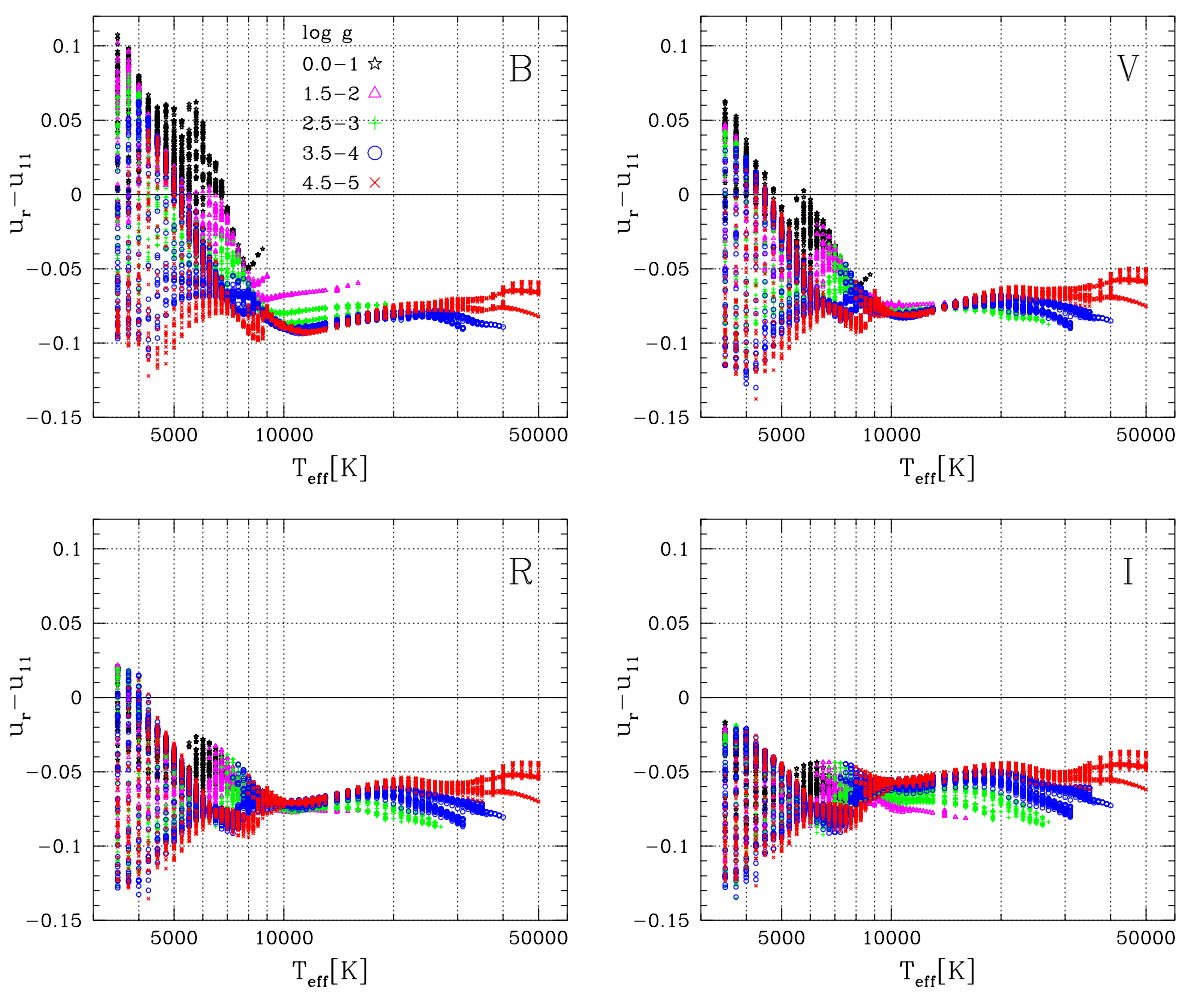

Figure 3: Difference between linear coefficients $u_{r}$ and $u_{11}$ for the full range of models, plotted as a function of $T_{e f f}$ for $B V R I$ bands as marked in the panels, color-coded by surface gravity $\log g$ (see top left).

Figure 5, with the first term marked in red. The PCA LD coefficient ranges from $\kappa_{P K}=-0.1620$ for the most peaked profile to $\kappa_{F L}=0.0902$ for the flattest. Returning to Table 1 and the right column of Figure 2, we see that the 2-term PCA model has an average intensity residual lower than $0.5 \%$ and average flux excess lower than $0.07 \%$.

\section{Microlensing Event Analysis: OGLE 2004-BLG-254}

Single-lens gravitational microlensing of different PCA LD shapes is illustrated in Figure 5 for a source radius $\rho_{*}=0.1$ Einstein radii. The amplification as a function of source-center position

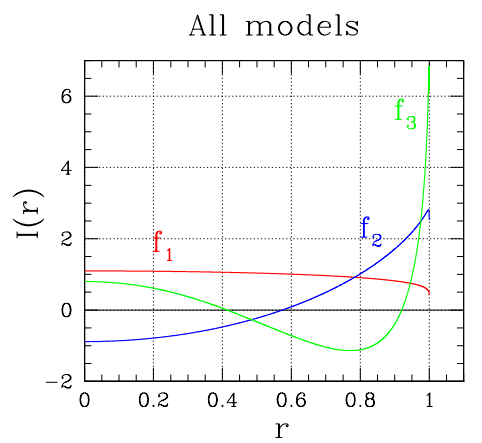

Bulge microlensing

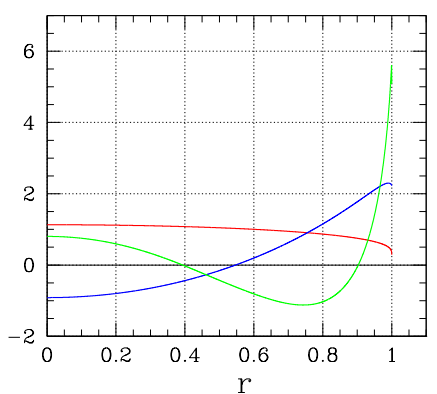

9 cool giants

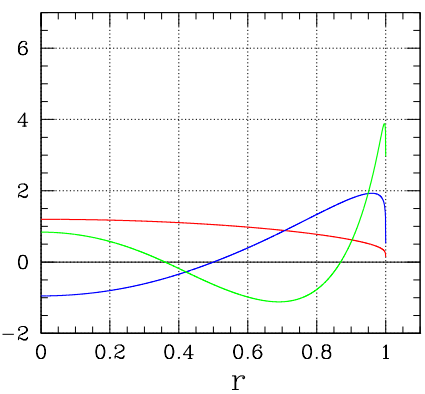

Figure 4: First three functions of LD bases obtained by principal component analysis of $B V R I$ profiles of the full Kurucz grid (left), of a subset more relevant for microlensed sources in the Bulge (center; VRI profiles, $\left.T_{e f f}=3500 \ldots 7000 \mathrm{~K}, \log g=0 \ldots 4,[\mathrm{Fe} / \mathrm{H}]=-2 \ldots 1, v_{t}=2 \mathrm{~km} \mathrm{~s}^{-1}\right)$, and of a set of BVRI profiles of 9 cool giants (right; $\left.T_{e f f}=3500 \ldots 4000 \mathrm{~K}, \log g=0 \ldots 1,[\mathrm{Fe} / \mathrm{H}]=0, v_{t}=2 \mathrm{kms}^{-1}\right)$. 

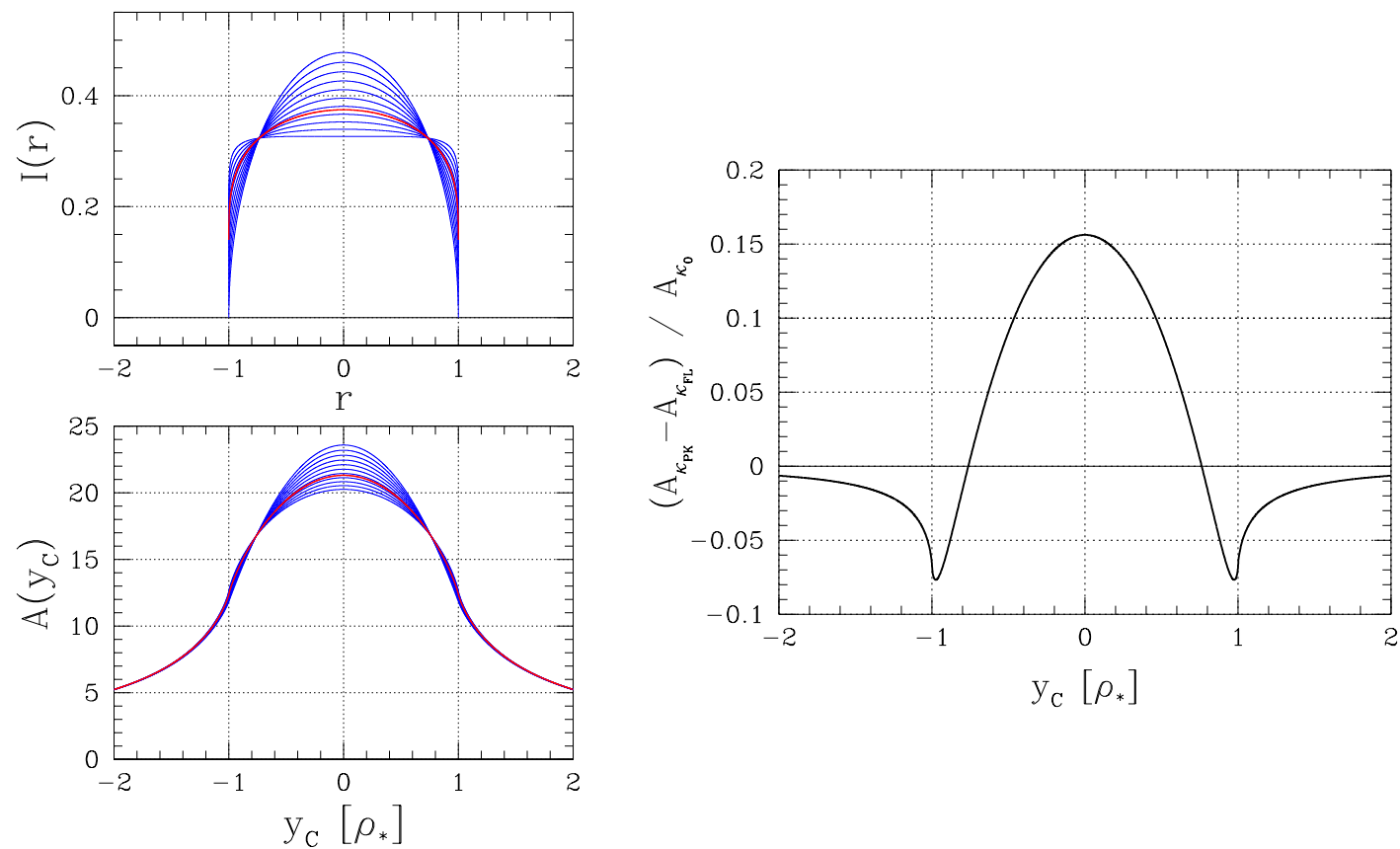

Figure 5: Point-lens microlensing of a source with radius $\rho_{*}=0.1$ with the 2-term PCA LD model. Top left: LD profiles. Bottom left: corresponding microlensing amplification $A$ for different source-center displacements $y_{c}$ from the lens. Right: microlensing chromaticity of the PCA LD model as a function of $y_{c}$.

$A\left(y_{c}\right)$ is plotted in the bottom left panel, with the LD profiles aligned in the top left panel. The relative sensitivity to LD variations may be measured by the chromaticity of the PCA LD model, obtained by taking the difference between the amplifications of the most centrally peaked $A_{\kappa_{P K}}$ and the flattest $A_{\kappa_{F L}}$ profiles and dividing it by the amplification $A_{\kappa_{0}}$ of the first PCA term. The amplifications differ by more than $15 \%$ for a lens directly aligned with the source center, with a secondary peak exceeding $7 \%$ at the limb. For a source positioned $2 \rho_{*}$ from the lens the variation is already lower than $1 \%$. The chromaticity curve remains practically unchanged for smaller sources.

The single-lens event OGLE 2004-BLG-254 with a caustic-crossing of a K3 III bulge giant was analyzed in detail by Cassan et al. (2006), who already showed that the measured linear LD coefficients were in better agreement with the new coefficients, even though small discrepancies remained. Here we concentrate on LD measurement, testing both the linear and PCA LD models. We fitted four $I$-band light curves within \pm 20 days of the peak using common global parameters

\begin{tabular}{|l|c|c|}
\cline { 2 - 3 } \multicolumn{1}{c|}{} & Linear LD coefficient & PCA LD coefficient \\
\hline Boyden & $0.840_{-0.064}^{+0.092}$ & $-0.1156_{-0.0464}^{+0.0091}$ \\
OGLE & $0.605_{-0.105}^{+0.062}$ & $-0.0459_{-0.0156}^{+0.0148}$ \\
SAAO & $0.624_{-0.084}^{+0.069}$ & $-0.0498_{-0.0144}^{+0.0305}$ \\
UTas & $0.643_{-0.091}^{+0.096}$ & $-0.0559_{-0.0358}^{+0.0235}$ \\
\hline Kurucz range & $(0.580,0.645) r$-int. & $(-0.0489,-0.0299)$ \\
& $(0.620,0.683) 11-$ pt. & \\
\hline
\end{tabular}

Table 2: LD coefficients of the OGLE 2004-BLG-254 source star measured from four I-band light curves. Ranges of coefficients of 45 plausible Kurucz grid models are listed at bottom (parameters $\left.T_{\text {eff }}=4000 \ldots 4500 \mathrm{~K}, \log g=1.5 \ldots 2.5,[\mathrm{Fe} / \mathrm{H}]=0 \ldots 0.5, v_{t}=2 \mathrm{kms}^{-1}\right)$. 

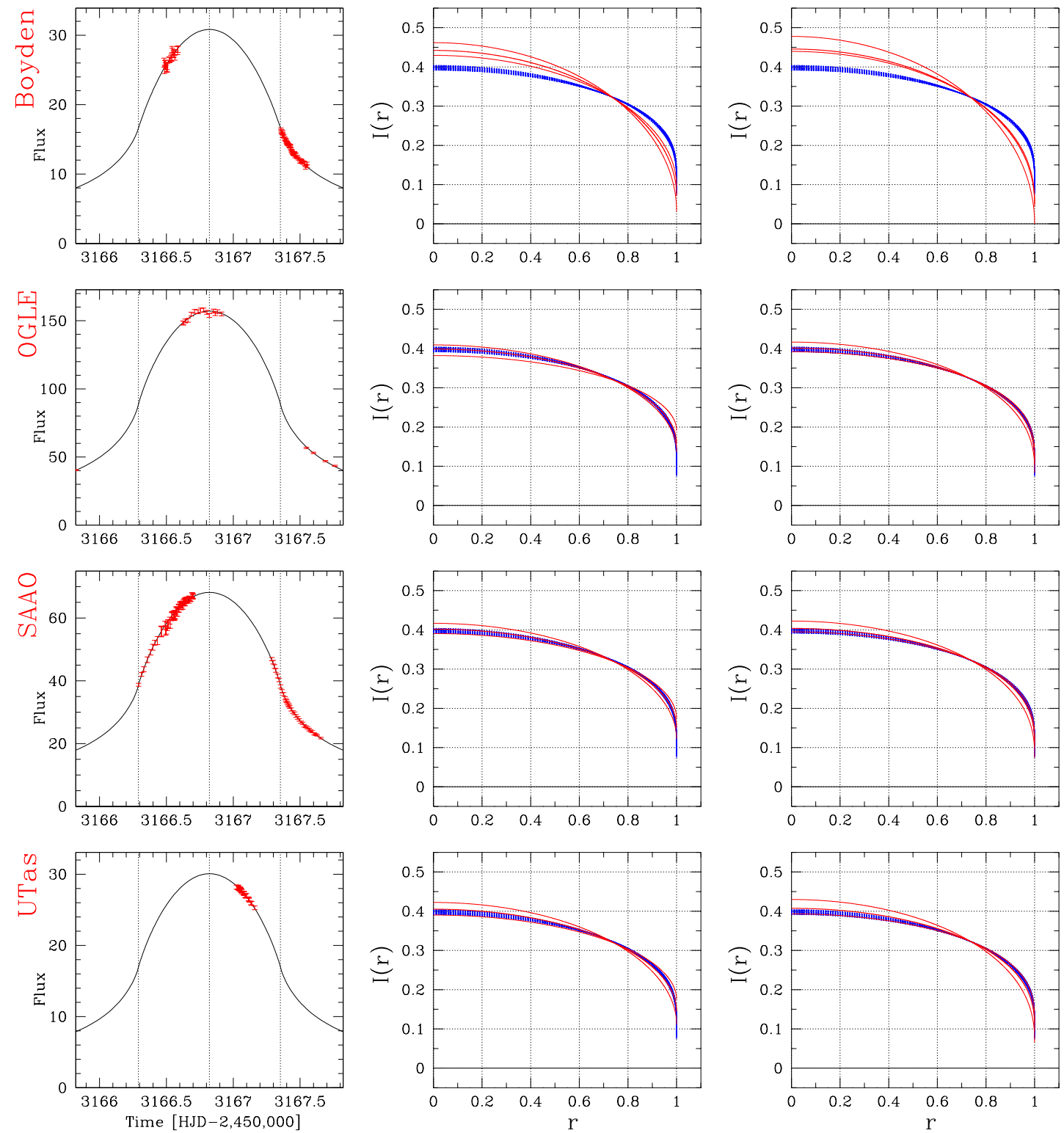

Figure 6: Four $I$-band light curves of OGLE 2004-BLG-254 and their measured LD profiles. Left: lightcurve peak in arbitrary flux units; dotted lines mark limb-crossings and closest approach $\left(0.14 \rho_{*}\right)$. Middle: measured linear LD (bold red) normalized to unit flux with $1 \sigma$ error bars (thin red), plotted with 45 nonapproximated plausible Kurucz profiles (dotted blue). Right: measured PCA LD with same Kurucz profiles.

(impact parameter $u_{0}$, source radius, peak time $t_{0}$, and Einstein-radius crossing time $t_{E}$ ) and individual blended flux and two parameters of the LD profile. Interestingly, the main improvement came from omitting a fifth light curve from the analysis, which had many points during the crossing but with a large scatter and possible systematic problems. The high number of its points in comparison with the fewer points in the other curves skewed the global parameters of the original results and led to the limb-darkening discrepancies.

The values of the global parameters obtained using the PCA model are $u_{0}=5.7_{-1.5}^{+1.7} \times 10^{-3}$, $\rho_{*}=4.010_{-0.011}^{+0.067} \times 10^{-2}, t_{E}=13.38_{-0.08}^{+0.25} d$, and $t_{0}=3166.8222_{-0.0017}^{+0.0014} d$. The measured LD co- 
efficients for the two models are summarized in Table 2 together with the respective ranges of theoretical values for a small grid of plausible Kurucz models of the source. We first see that all light curves except Boyden give mutually compatible results. The Kurucz values also show a better agreement of the measured values with the new linear coefficients than with the 11-point fit values. Figure 6 includes details of the light-curve peaks and the measured linear LD and PCA LD profiles (in red), normalized to unit flux and plotted together with similarly normalized non-approximated profiles of the plausible Kurucz models (in blue). The Boyden results are affected by the absence of baseline points in the light curve (no points outside the plotted detail), which leads to an overestimated blend and a too peaked LD profile. For the other light curves the agreement with the theoretical profiles is excellent.

When comparing the PCA and linear LD results, a closer inspection of the lower three measurements reveals an interesting difference. The PCA results all have consistently the same position relative to the Kurucz profiles. In fact, the best-fit PCA profiles all point to the cooler end of the included Kurucz model parameter range, in agreement with the source analysis by Cassan et al. (2006). The LLD model results for the OGLE light curve are flatter than for the other two light curves, and point to somewhat warmer Kurucz models. These results, which can be further refined, already indicate that the PCA model may provide more robust LD measurement.

\section{Conclusions}

When using simple analytical LD laws, one should be aware that tabulated coefficients are often biased and do not necessarily give the best-fitting version of the given law. The procedure introduced in Heyrovský (2007) shows how to obtain better coefficient values. For analysis of observational data, the PCA approach to LD modeling provides an accurate and flexible alternative to the often inadequate analytical laws.

\section{Acknowledgments}

I thank Arnaud Cassan, Jean-Philippe Beaulieu and the PLANET team for providing the data of OGLE 2004-BLG-254. This work was supported by Czech Science Foundation grant GACR 205/07/0824 and by the Ministry of Education, Youth and Sports grant MSM0021620860.

\section{References}

[1] Cassan, A., et al. 2006, A\&A, 460, 277

[2] Claret, A. 2000, A\&A, 363, 1081

[3] Díaz-Cordovés, J., Claret, A., \& Giménez, A. 1995, A\&AS, 110, 329

[4] Heyrovský, D. 2003, ApJ, 594, 464

[5] Heyrovský, D. 2007, ApJ, 656, 483

[6] Kurucz, R. L. 1993a, Limbdarkening for $2 \mathrm{~km} / \mathrm{s}$ grid (No. 13): [+1.0] to [-1.0] (Cambridge: SAO)

[7] Kurucz, R. L. 1993b, Limbdarkening for $2 \mathrm{~km} / \mathrm{s}$ grid (No. 13): [+0.0] to [-5.0] (Cambridge: SAO)

[8] Kurucz, R. L. 1994, Solar abundance model atmospheres for 0,1,2,4,8 km/s (Cambridge: SAO) 